\title{
Tobramycin Nebulizer Solution in Severe COPD Patients Colonized with Pseudomonas Aeruginosa: Effects on Bronchial Inflammation
}

In the above article by Roberto Dal Negro et al., published in the October 2008 issue of Advances in Therapy, Dompé Farmaceutici was incorrectly listed as the sponsor. Dompé Farmaceutici would like to state that they are not the sponsor or supporter of the study, and did not support the authors in any way. 